\title{
Erratum: Quantitative high throughput screening using a primary human three-dimensional organotypic culture predicts in vivo efficacy
}

Hilary A. Kenny, Madhu Lal-Nag, Erin A. White, Min Shen, Chun-Yi Chiang, Anirban K. Mitra, Yilin Zhang, Marion Curtis, Elizabeth M. Schryver, Sam Bettis, Ajit Jadhav, Matthew B. Boxer, Zhuyin Li, Marc Ferrer \& Ernst Lengyel

Nature Communications 6:6220 doi: 10.1038/ncomms7220 (2015); Published 5 Feb 2015; Updated 3 Feb 2016

The data plotted in the graph on the right in Fig. $2 \mathrm{~b}$ of this Article were inadvertently duplicated in the adjacent graph during the production process. The correct version of Fig. 2 appears below.
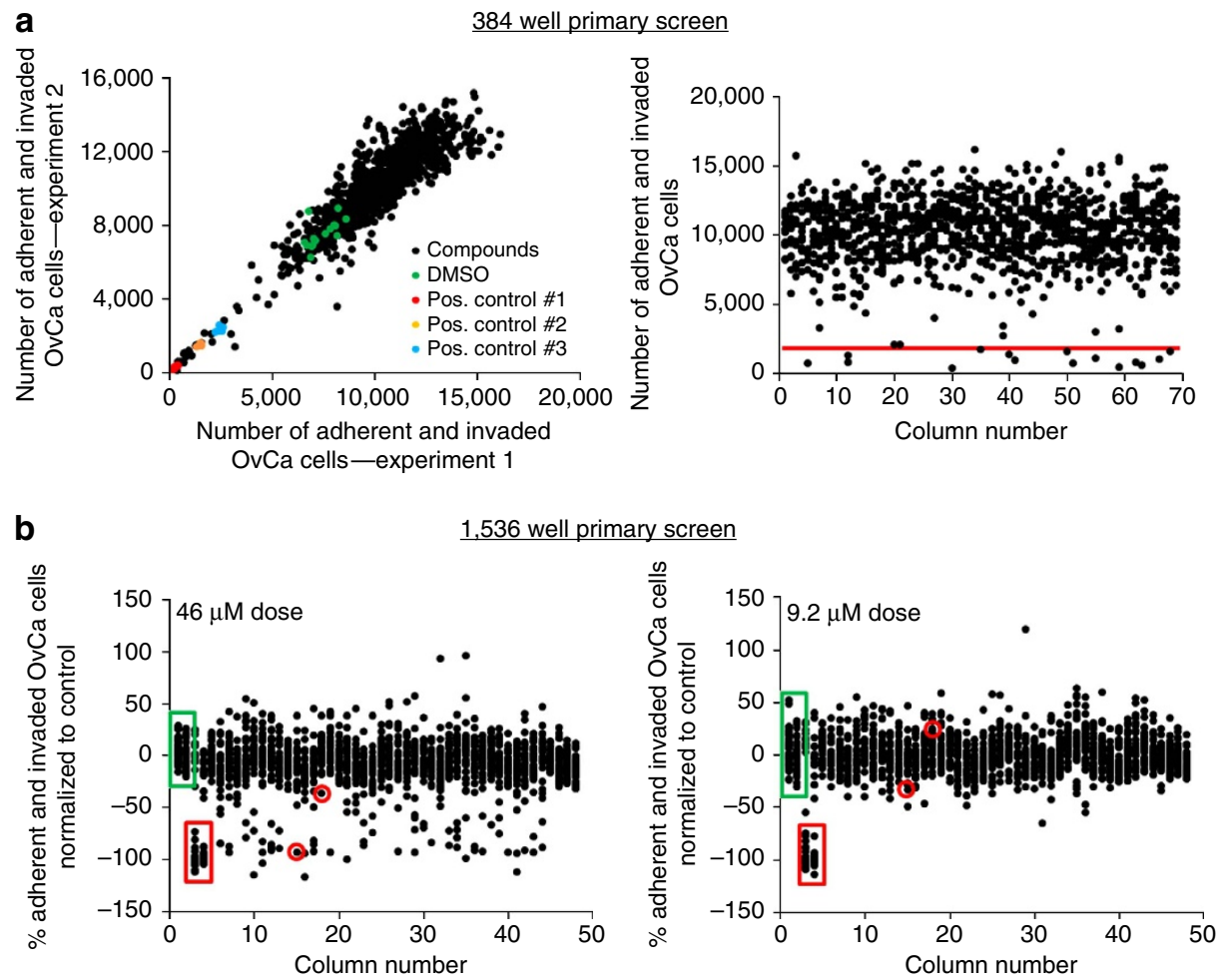

Figure 2 | 